\title{
Kernos
}

Revue internationale et pluridisciplinaire de religion grecque antique

8 | 1995

Varia

\section{Athéna chez Homère ou le triomphe de la déesse}

\section{Paul Wathelet}

\section{CpenEdition}

\section{Journals}

Édition électronique

URL : http://journals.openedition.org/kernos/601

DOI : 10.4000/kernos.601

ISSN : 2034-7871

\section{Éditeur}

Centre international d'étude de la religion grecque antique

\section{Édition imprimée}

Date de publication : 1 janvier 1995

Pagination : 167-185

ISSN : 0776-3824

\section{Référence électronique}

Paul Wathelet, « Athéna chez Homère ou le triomphe de la déesse », Kernos [En ligne], 8| 1995, mis en ligne le 11 avril 2011, consulté le 01 mai 2019. URL : http://journals.openedition.org/kernos/601 ; DOI : 10.4000/kernos.601 
Kernos, 8 (1995), p. 167-185.

\section{Athéna chez Homère ou le triomphe de la déesse}

Dans l'Iliade, Athéna est constamment l'alliée des Achéens contre les Troyens. Dès le premier chant, quand Achille, furieux, veut tuer Agamemnon, elle se précipite pour arrêter son geste (A 195). Lorsque, au chant II, Agamemnon annonce à l'assemblée son intention de rentrer à Argos, Athéna fait intervenir Ulysse et elle arrête le mouvement (B 163). Plus loin (B 446), elle entraîne les Achéens en brandissant l'égide aux franges d'or et en leur inspirant le goût de la bataille. Lors du duel qui les oppose, elle soutient Ménélas contre Pâris $(\Delta 8)$ et elle murmure contre Zeus qui suggère la paix ( $\Delta$ 20). Elle provoque la rupture de la trêve et incite Pandaros ${ }^{1}$ à frapper Ménélas ${ }^{2}$, mais elle fait en sorte que sa blessure soit plus spectaculaire que grave $(\Delta 73$ 147). Elle mène l'armée achéenne contre celle des Troyens conduits par Arès ${ }^{3}$ ( $\Delta$ 439). Au chant $V$, elle écarte Arès du combat afin qu'il ne puisse aider les Troyens «qui plient sous les Danaens» (E 37). Tout au long de l'aristie de Diomède, elle assiste le fils de Tydée. Au chant VI, elle refuse le voile somptueux qu'Hécube et les dames de Troie viennent lui offrir dans son temple à Ilion ${ }^{4}$ ( $Z$ 237-311). Au chant VII, pour couvrir les Achéens qui cèdent du terrain, elle persuade Apollon d'arrêter le combat afin d'organiser un duel entre Hector et un héros achéen, ce sera Ajax, fils de Télamon ${ }^{5}$. Quand, plus loin, Zeus interdit aux dieux de participer à la bataille, elle plaide pour les Argiens $(\Theta 30-37)$. Elle tente ensuite de passer outre à l'ordre du Cronide, mais ce dernier envoie Iris pour l'arrêter.

1 Sur Pandaros, cf. P. WATHELET, Dictionnaire des Troyens de liliade, Université de Liège, 1988, p. $799-809, n^{\circ} 260$ IIáv8apos.

2 Sur Ménélas, proche d'Arès, cf. P. WATHELET, Arès chez Homère ou le dieu mal aimé, in LEC, 60 (1992), p. 113-128, sp. p. 122-123.

3 Sur le rôle d'Arès dans l'Iliade, cf. P. WATHELET, $t$ bid.

4 Mal inspirée, Hécube a choisi un voile " œuvre des Sidoniennes, qu'Alexandre pareil aux dieux a ramené de Sidon, ... au cours du même voyage dont il a ramené aussi Hélène ... » (Z 289292). Rappel indiscret du jugement de Pâris dont Athéna ne doit pas avoir gardé un très bon souvenir (cf. plus loin, n. 10).

5 Le duel entre Hector, héros qui attaque, et Ajax, fils de Télamon, héros qui défend, est en fait un vieux thème épique, bien antérieur à Homère et qui remonte sans doute à une époque où les deux personnages étaient des héros grecs. Le duel est bien attesté dans l'iconographie archaïque et il revient en plusieurs passages de l'Iliade. Cf. P. WATHELET, Dict. des Troyens, op. cit. (n. 1), p.

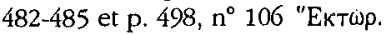


Dans la Dolonie ${ }^{6}$, elle entend les prières de Diomède et d'Ulysse, elle leur envoie un présage favorable ${ }^{7}$ et elle retient le fils de Tydée quand il veut s'emparer du char de Rhésos. Au chant XI, avec Héra, elle salue Agamemnon d'un coup de tonnerre $(\Lambda 45)^{8}$, puis elle évite que la blessure infligée par Sokos à Ulysse ne soit mortelle $(\Lambda 437)^{9}$. Au chant XV, Zeus aide Hector dont la vie sera brève, puisque, déjà, «Athéna pousse vers lui le jour où il doit succomber » (O 614). Elle stimule les guerriers achéens qu'exhorte Nestor $(O$ 668). Au chant XVII, elle est envoyée par Zeus pour animer la bataille, elle excite les Danaens (P 544). Quand, privé de ses armes, Achille veut chasser les Troyens, elle jette sur ses épaules l'égide frangée et elle lui confère une apparence extraordinaire $(\Sigma 203)$, puis elle pousse un cri en même temps que le Péléide $(\Sigma$ 217). Malgré l'avis de Polydamas, Hector et les Troyens poursuivent la lutte, parce qu'« Athéna leur a ravi la raison» $(\Sigma 311)$. Au chant suivant, elle met le nectar et l'ambroisie dans le cour d'Achille, afin qu'il ne souffre pas de la faim. Dans la Théomachie ( $\Upsilon$ 33), elle est évidemment du côté des Achéens. Plus loin ( $\Upsilon$ 438), elle détourne d'Achille la pique d'Hector. Au chant suivant, elle réconforte le Péléide et lui met la force au cœur, quand il se bat contre les fleuves ( $\Phi$ 304), puis elle lutte victorieusement contre Arès $(\Phi$ 391-392). Elle trompe Hector pour qu'il affronte Achille, lors de leur ultime duel et elle aide Achille à tuer le Priamide (X 188-305).

Le début du dernier chant de l'fliade donne une explication à l'attitude de la déesse : son hostilité, comme celle d'Héra, à l'égard des Troyens date du jugement de Pâris $(\Omega 25-30)^{10}$. Athéna n'a pas toujours manifesté de l'hostilité aux Troyens. Une génération auparavant, elle les avait aidés à construire un rempart de terre pour aider Héraklès à échapper à l'attaque du monstre marin envoyé par Poseidon ( $\Upsilon 146)$, mais ceci se passait avant le fameux jugement.

En somme, dans l'lliade, Athéna tient du côté achéen le rôle qu'Apollon joue chez les Troyens ${ }^{11}$. L'action de la déesse est toutefois très différente. Le fils de Létô aide régulièrement les Troyens, mais il reste un dieu distant, qui se manifeste avec vigueur, de manière ponctuelle, mais sans poursuivre

6 Cf. P. WATHELET, Rbésos ou la quête de l'immortalité, in Kernos, 2 (1989), p. 213-231.

7 Ce présage est le cri d'un héron ( $(\varepsilon \omega \omega \delta$ เós) entendu à droite de leur chemin. Le héron reparaît dans un fragment des Psychagogoi d'Eschyle, dans la prédiction que Tirésias fait à Ulysse de sa mort (F 275, 1. 1, St. RADT). Il n'est pas impossible que l'oiseau constitue une annonce de l'entrée dans l'Autre Monde, ce qui serait conforme à l'ensemble de la Dolonie.

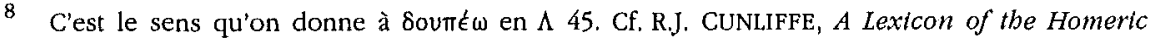
Dialect, Londres, 1924 [Oklahoma Press, 1963], p. 98-99.

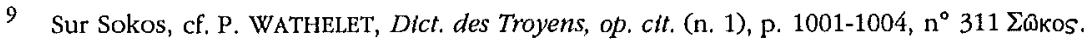

10 C'est la seule mention du fameux jugement dans l'épopée homérique et les vers étaient considérés comme inauthentiques par Aristarque, pour des raisons philologiques, ce qui impressionne. (Cf. A. SEVERYNS, Le Cycle éplque dans l'École d'Aristarque, Liège-Paris, 1928, p. 261-264). Même si le passage n'est pas authentique, il est quasi assurê qu'Homère connaissait l'épisode.

11 Cf. P. WATHELET, Apollon dans /liade ou le protecteur des Troyens, in Minerva, 7 (1993), p. $57-77$. 
longtemps son effort. Athéna, elle, s'acharne et prend une part directe à l'action. Son intervention dans l'aristie de Diomède est caractéristique à cet égard. La déesse n'a cessé d'aider le fils de Tydée. Elle trouve que le cocher du héros, Sthénélos, n'est pas assez efficace. Elle le bouscule et le fait tomber, pour saisir les rênes et prendre sa place ${ }^{12}$. Son attitude est d'autant plus remarquable que le rôle de cocher est dans l'Iliade une fonction socialement inférieure, indigne a priori d'une divinité ${ }^{13}$. Pour Athéna, la fin justifie les moyens.

Non seulement l'action de la déesse est constante et immédiate, mais, loin d'être impulsive comme Apollon, elle emploie régulièrement la ruse. C'est le cas au chant VII : alors que les Troyens dominent et pour faire diversion, elle persuade Apollon d'arrêter le combat et d'organiser le duel qui n'aboutira à rien : il sera arrêté par les hérauts, en raison de l'arrivée de la nuit. L'activité d'Athéna est aussi empreinte de prudence : dans la Dolonie, après avoir encouragé Diomède et Ulysse dans leur entreprise, elle arrête le fils de Tydée quand il veut s'emparer du char de Rhésos (K 503-511) ${ }^{14}$.

L'Iliade fait allusion à quelques autres activités d'Athéna qui ne sont pas étroitement liées aux épisodes de la guerre de Troie racontés dans le poème. Au temps passé, la déesse est intervenue pour secourir Nestor $(H 154$ et $\Lambda$ 721) ou, comme elle le rappelle à Héra, elle a souvent sauvé Héraklès ( $\Theta$ 362), elle a assisté Achille lorsqu'il prenait Lyrnessos ( 191 ). Les attributions d'Athéna ne sont pas seulement guerrières. Elle patronne tout ce qui demande de l'intelligence pratique. Une comparaison évoque un charpentier expert, qui « connaît son art à fond par l'intervention d'Athéna » $(\mathrm{O}$ 410-411). Un passage du chant $\mathrm{V}$ raconte la mort, sous les coups de Mérion, de Phéréklos qu'Athéna chérissait, car, bon charpentier, il avait construit les vaisseaux d'Alexandre (E 59$68)^{15}$. La déesse s'occupe aussi des travaux proprement féminins et qui demandent ingéniosité et habileté. Dans la scène des $\Lambda$ เтa ${ }^{16}$, quand il refuse les propositions d'Agamemnon, spécialement celle qui consiste à lui offrir la main de la plus belle de ses filles, le Péléide précise «le disputât-elle pour la beauté à Aphrodite d'or, pour les travaux à Athéna aux yeux pers » (I 390 trad. P. Mazon).

12 Sous le poids de la déesse, l'essieu de chêne du char gémit (E 838-839), détail qui ne correspond plus à notre sens de la beauté féminine.

13 Cf. P. WATHELET, Dict. des Troyens, op. clt. (n. 1), p. 807-808, $\mathrm{n}^{\circ} 260$ Máv8apos.

14 Sur la valeur symbolique de ce char et sur son importance, cf. P. WATHELET, Rbésos..., art. cit. (n. 6), p. 226-229.

15 Cf. P. WATHELET, Dict. des Troyens, op. clt. (n. 1), p. 1046-1049, n 334 ФÉ $\rho \in k \lambda o s$.

16 Sur l'importance capitale de cette scène dans l'ensemble de l'Iliade, cf. D. AUBRIOT, Remarques sur le cbant $I X$ de liliade, in BAGB (1985), p. 257-279; Remarques sur le personnage de Pbénix au chant $L X$ de l Iliade, in $B A G B(1984)$, p. 339-362. 
Enfin, Athéna est une déesse poliade qui protège les cités. À Troie, elle possède un temple qui domine la ville $(\mathrm{Z} 297-299)^{17}$ et, au moment où le Catalogue des Vaisseaux mentionne le contingent athénien, il fait une courte digression qui rappelle qu'Athéna a nourri Érechthée, fils de la Terre, qu'« elle l'a installé dans son riche sanctuaire. Aussi les Athéniens lui offrent-ils là taureaux et agneaux à chaque retour de l'armée » $(\mathrm{B} 547-551 \text { - trad. P. Mazon })^{18}$.

Les dieux du panthéon grec ne cessent de s'opposer dans la mesure où leurs sphères d'influence se recoupent partiellement. Comme ces dieux sont immortels, les conflits le sont aussi et l'ensemble forme une manière de système constamment en équilibre instable. On ne peut donc étudier une divinité particulière qu'au travers des relations qu'elle entretient avec les autres dieux.

L'attitude d'Athéna à l'égard de Zeus est variable. La déesse témoigne en apparence d'une grande soumission à son père. Elle cède sa place à Thétis qui vient s'asseoir auprès de Zeus $(\Omega 100)$ et en général elle semble très liée au Cronide. Il n'empêche que, parfois, elle le critique. L'accusant d'ingratitude puisqu'elle a sauvé son fils Héraklès ( $\Theta$ 364-369), elle propose à Héra de reprendre le combat pour défendre les Achéens. Elles sont toutes deux en route vers la plaine, quand le Cronide s'en aperçoit et leur donne l'ordre formel de s'arrêter. Impressionnées, les deux déesses obéissent sur la suggestion d'Héra. Zeus se moque d'elles, alors qu'à l'écart elles témoignent de leur mauvaise humeur $(\Theta 350-460)$. L'Iliade conserve le souvenir d'une révolte des dieux contre Zeus. Au chant I (A 398), Thétis rappelle que, seule, elle a défendu Zeus contre les Olympiens qui voulaient l'enchaîner; trois membres du

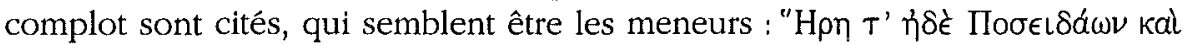

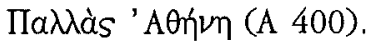

En plusieurs passages de l'Iliade, Athéna et Héra agissent de concert. Leur bonne entente correspond à une communauté d'intérêt depuis le jugement de Pâris. Héra, femme de Zeus, est une déesse « assise » xpvoóopovos (A 611, 0 5 , etc.), qui voit les choses de haut et charge Athéna d'intervenir directement. C'est elle qui, au premier chant, dépêche Pallas afin qu'elle arrête Achille dans son geste. Au deuxième chant, quand, maladroitement, Agamemnon propose à ses troupes de rentrer, pour tester leur pugnacité, et qu'elles le prennent au

17 On a contesté l'authenticité du passage sous le prétexte que les temples étaient inconnus à l'époque homérique, l'argument est aujourd'hui sans valeur (cf. G.S. KIRK, The Illad: A Commentary. II, 5-8, Cambridge Univ. Press, 1990, p. 104, en E 445-8). Homère attribue aux Troyens les croyances et les pratiques religieuses des Grecs, ce qui procède d'un mouvement propre au développement de la matière épique. Hécube et les dames troyennes partant en procession au temple d'Athéna pour lui porter un voile font penser à la procession des Panathénées à Athènes.

18 Dans le passage du Catalogue des vatsseaux consacré à l'Attique, une seule ville est mentionnée, Athènes, alors que l'épopée homérique connaît les noms d'autres localités dont Marathon ( $\eta$ 80) et Thorikos ( $H$. a Dém., 126). La place qu'occupe la liste des localités dans les autres contingents est prise par la présente digression. On peut se demander si cette digression n'a pas été introduite dans le morceau par un aède qui connaissait le synécisme athénien et qui voulait l'inscrire dans un passage aussi prestigieux que le Catalogue des vaisseaux. 
mot, c'est Héra qui envoie Athéna redresser la situation. Alors qu'au chant IV, la trêve est signée, Héra obtient de Zeus qu'il enjoigne à Athéna de pousser les Troyens à la rompre. La déesse s'empresse d'obéir et arrive si brusquement

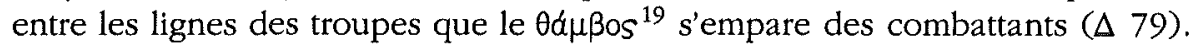
$\mathrm{Au}$ chant V, Héra et Athéna descendent dans la plaine, avec l'accord de Zeus, pour aider les Achéens contre Arès (E 711-793). Au chant VIII, les deux déesses tentent à nouveau d'intervenir, mais, malgré l'ordre de Zeus, comme on l'a rappelé plus haut ( $\Theta$ 350-488). Dans son discours à Achille, Ulysse rappelle les conseils que Pélée donnait à son jeune fils : «la victoire, ce sont Athéna et Héra qui te l'octroieront, si elles le veulent » (I 254-255). Alors qu'Énée court le risque d'être tué par Achille, Poseidon s'emploie à le sauver, mais Héra et Athéna refusent catégoriquement de secourir un Troyen $(\Upsilon 297-317)^{20}$. Héra et Athéna sont parfois associées à Poseidon. Tous trois se sont révoltés contre Zeus (A 400), tous trois se tiennent du côté des Achéens ( $\Upsilon$ 33). Les trois divinités sont de nouveau ensemble pour observer le duel entre Énée et Achille ( 2 291-317). Quand, au chant XI, il rappelle ses exploits passés, Nestor signale qu'il a fait une offrande à Athéna et à Poseidon $(\Lambda$ 728-729). Les deux divinités se retrouvent au chant XXI ( $\Phi$ 284) pour soutenir le combat d'Achille contre le Scamandre et le Simoïs. Toutefois, l'entente entre Poseidon et Athéna est loin d'être constante. Déjà dans l'Iliade, Athéna ne marque aucune sympathie à Ajax, fils d'Oilée, or les Ajax sont protégés de Poseidon ${ }^{21}$. Mais c'est surtout dans l'Odyssée que l'opposition entre les deux divinités éclatera.

Les relations entre Athéna et Apollon, Arès ou Aphrodite sont presque constamment conflictuelles, puisque ces trois divinités sont des défenseurs attitrés des Troyens. Avec Apollon, qui est à plusieurs reprises mentionné dans le même vers avec Zeus et Athéna (B 371,. H 132, II 97), l'opposition ne débouche jamais sur un combat, mais elle prend une forme indirecte : voulant provoquer la rupture de la trêve, Athéna suggère à Pandaros, un archer proche par le fait même d'Apollon, de tirer une flèche sur Ménélas en promettant au dieu une hécatombe d'agneaux nouveau-nés ( $\Delta$ 101). Quand le combat a repris, Apollon excite les Troyens, il n'en faut pas plus pour qu'Athéna entraîne les Achéens ( $\Delta 514)$. Au chant VII, comme on l'a vu, Athéna s'entend avec Apollon pour arrêter le combat et organiser un duel, auquel ils assistent déguisés en vautours (H 58). Dans la Dolonie, Athéna retient Diomède, quand

19 A. MOTTE, L'expression du sacré dans la religlon grecque, in L'expression du sacré dans les grandes religions, III, Louvain-la-Neuve, 1986, p. 170-172. - D. AUBRIOT, Remarques sur l'usage de $\theta a ́ \mu \beta o s$ et des mots apparentés dans /1liade, in Orpbeus, 2 (1989), p. 249-260.

20 Sur le passage, cf. P. WATHELET, Le mytbe d'Énée dans l'épopée bomérique. Sa survie et son exploitation poétique, in Mythe et Politique, Actes du Colloque de Liège 14-16 septembre 1989, Paris, Les Belles Lettres, 1990 (Btbl. de la Fac. de Pbil. et Lettres de l'Univ. de Liège, 257), p. 287-296.

21 Sur les deux Ajax, cf. P. WATHELET, Dict. des Troyens, op. cit. (n. 1), p. 249-250, $\mathrm{n}^{\circ} 18$ 'Akáuas II. 
Apollon s'aperçoit de ce qui se passe dans le camp des Thraces (K 516), Au moment où Hector va mourir, Apollon l'abandonne, alors qu'Athéna intervient.

À l'égard d'Arès, l'hostilité d'Athéna est tout aussi intense, mais, comme il s'agit d'un dieu peu estimé, la déesse prend moins de précautions. C'est sur un ton moqueur et méprisant qu'elle l'affronte au cours de la Tbéomacbie ( $\Phi$ 391415) et qu'elle le fait tomber de tout son long, au point que son corps monstrueux couvre sept plèthres. Même sa bienveillance apparente à l'égard du dieu est intéressée, elle le détourne du combat et elle l'amène sur les bords herbeux du Scamandre (E 29). Ce n'est pas pour une partie de campagne: «les Troyens alors plient sous les Danaens »... Ailleurs, au chant V, sous l'impulsion d'Athéna, Diomède finit par blesser Arès ( $E$ 846-863). Il est cependant des passages où les deux divinités sont réunies par une sorte de solidarité guerrière : c'est le cas sur le bouclier d'Achille $(\Sigma 516)^{22}$ ou bien quand Arès veut venger la mort de son fils Askalaphos (O 124), malgré l'ordre de Zeus. Athéna le retient, car elle craint pour les Olympiens.

La haine d'Athéna pour Aphrodite est totale, mais elle a peu l'occasion de se manifester directement: Aphrodite fréquente peu le champ de bataille. Toutefois, quand, lors de l'aristie de Diomède, la déesse veut sauver son fils Énée qui se trouve en grand danger, Athéna encourage le fils de Tydée à frapper la déesse. Blessée, celle-ci s'enfuit sur l'Olympe où elle est réconfortée par Zeus et par sa mère Dioné (E 297-430) ${ }^{23}$. Plus loin, au cours de la Théomacbie, Héra encourage Athéna à frapper Aphrodite qui veut secourir Arès. Pallas ne se le fait pas dire deux fois : d'un coup violent frappé à la poitrine, Aphrodite est projetée à terre, où elle vient retrouver Arès ( $\Phi$ 419433).

Les passages de l'Iliade qui viennent d'être évoqués montrent la victoire d'Athéna et l'importance du rôle de la déesse. On a réservé un passage où son succès apparaît spécialement et qui n'est pas guerrier. Il s'agit des jeux funèbres en l'honneur de Patrocle ${ }^{24}$. Ces jeux sont longuement racontés au chant XXIII, ils comportent les épreuves suivantes : la course de chars, le pugilat, la lutte, la course à pied, le combat, le lancer du disque, le tir à l'arc et le lancer de la javeline. Les protégés d'Athéna l'emportent au moins dans cinq épreuves sur huit et dans la principale, la course de chars, qui est beaucoup plus longuement décrite (près de quatre cents vers). Elle oppose Eumèle, fils

22 C'est la seule scène guerrière qui figure sur le Bouclier d'Achille, où Héphaïstos a multiplié les représentations pacifiques et qui, contrairement à l'usage du temps, n'ont rien d'effrayant. Cf. P. WATHELET, Le Bonclier d'Achille ou le pactfisme d'Homère, Université de Liège, Entretiens sur l'Antiquité gréco-romaine, 1995.

23 V. PIRENNE-DELFORGE, L'Apbrodite grecque, Athènes-Liège, CIERGA, 1994 (Kernos, suppl. 4), p. 311-312.

24 J.-P. VERNANT - M. DETIENNE, La Métis d'Antiloque, in REG, 80 (1967), p. 68-83. - Sur l'ensemble du passage et les autres jeux funèbres mentionnés dans l'épopée homérique, cf. N. RICHARDSON, The Iliad: A Commentary. Volume VI : books 21-24, Cambridge Univ. Press, 1993, p. 201-203. 
d'Admète, Diomède, Ménélas, Antiloque et le Crétois Mérion. Certains des attelages en présence sont de haute qualité. Venu de Thessalie, région célèbre pour ses élevages de chevaux, Eumèle part favori ${ }^{25}$. Diomède possède les coursiers merveilleux de Tros qu'il a enlevés à Énée, lors de son aristie, racontée au chant $\mathrm{V}^{26}$. Ménélas aligne son propre cheval Podargos « aux pieds blancs » et Aithé ${ }^{27}$, une jument de grand prix qu'Agamemnon lui a prêtée. Les coursiers de Mérion ne semblent pas avoir de qualité particulière. Quant à Antiloque, si lui-même fait preuve de grandes qualités, il est affligé de chevaux dont Nestor reconnaît qu'ils sont de piètres coursiers. Plein d'expérience, Nestor conseille à son fils de compenser l'infériorité de son attelage par son adresse et par la ruse, au moment où, au bout de la carrière, il tournera autour de la borne afin de revenir au but ${ }^{28}$.

Après tirage au sort de la place des concurrents, le départ est donné. Les attelages s'élancent, ils contournent la borne et reviennent; Eumèle est le premier, mais il risque d'être dépassé par Diomède. Or Admète avait bien accueilli Apollon, lorsqu'il avait dû s'exiler sur terre après le meurtre du serpent Python. Le dieu veut favoriser le fils de son ancien bienfaiteur et, pour empêcher Diomède de le dépasser, il lui fait sauter le fouet des mains. Mais Athéna, protectrice de Diomède, veille. Elle rend son fouet à Diomède et rompt le joug qui tient l'attelage d'Eumèle : ses deux coursiers s'écartent, le timon du char heurte le sol, Eumèle tombe du char et il s'écorche fortement au contact de la terre. Diomède le dépasse, Athéna insuffle l'ardeur à ses chevaux et il gagne la course.

Comme dans d'autres circonstances, l'attitude des deux divinités est révélatrice de leur caractère : Apollon intervient le premier de manière impulsive, il rompt ainsi une sorte de convention qu'auraient pu observer les dieux de ne pas intervenir dans un concours entre mortels et où leur force est tellement supérieure qu'elle fausse le jeu. Une fois qu'il est intervenu, Apollon abandonne son protégé à son sort. Sa réaction amène Athéna, que l'on sent aux aguets ( $\Psi 388$ ), à réagir immédiatement et de manière décisive. Non seulement elle répare le dommage fait par Apollon en rendant son fouet à Diomède, mais elle inflige à Eumèle un coup irréparable, qui aura pour lui des conséquences beaucoup plus graves : sa course est perdue, il est jeté à terre et écorché.

25 Sur les chevaux d'Admète dont a hérité Eumèle, lls ont été élevés par Apollon (B 763-767) Faut-il s'interroger sur l'âge de ces juments ? Le temps mythique n'est pas le temps réel.

26 Tros avait reçu de Zeus des chevaux mervelleux, en compensation de l'enlèvement de Ganymède (E 222-3). En cachette de Laomédon, Anchise avait fait saillir ses juments par ces chevaux extraordinaires, six poulains en sont nés (E 265-272). Énée en avait reçu deux, ils tiraient son char quand, au chant $\mathrm{V}$, il a affronté Diomède en compagnie de Pandaros. Ce dernier a été tué et Énée blessé, Diomède s'est emparé des chevaux (E 319-327 - $\theta$ 105-111).

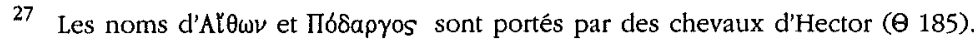

28 Cf. J.-P. VERNANT - M. DETIENNE, art. cit. (n. 24), et sur le discours de Nestor, R.P. MARTIN, The Language of Heroes. Speech and Performance in the Iliad, Ithaca, Comell Univ. Press, 1989, p. 108-109. 
Le cas d'Antiloque est plus curieux. Dès que Diomède a gagné et qu'Eumèle est hors course, Antiloque exhorte longuement ses chevaux. Il ne leur demande pas de lutter contre les étalons de Diomède que protège Athéna, - Antiloque est prudent - mais il les presse de courir plus vite que ceux de Ménélas et spécialement qu'Aithé, « une femelle ». Si ses chevaux ne se hâtent pas, ils ne seront plus bien nourris, mais on les mettra à mort ${ }^{29}$. Les chevaux s'inquiètent « de la voix grondeuse de leur maître » et ils prennent de l'allure.

Comme son père le lui avait indiqué, mais pour un autre moment de la course, Antiloque aura recours à la ruse pour évincer Ménélas. Tout au long de l'épopée, Nestor et les siens sont protégés par Poseidon, mais, dans la mesure où il utilise la ruse, Antiloque est proche d'Athéna ${ }^{30}$, alors que Ménélas, lui, est "chéri d'Arès " ${ }^{31}$. Grâce à son intelligence et à son habileté, Antiloque arrive second après Diomède. Ménélas n'a pas eu le temps de rattraper le fils de Nestor et de le dépasser. Le quatrième est Mérion, personnage rapproché d'Enyalios et par conséquent, chez Homère, d'Arès ${ }^{32}$. Le dernier, totalement déconfit, est Eumèle.

Achille octroie le premier prix à Diomède et il veut donner le second à Eumèle, en manière de consolation, mais Antiloque proteste et défend son bon droit. Achille cède, il donnera un autre prix à Eumèle. Fils de Nestor, héros chthonien, Antiloque s'oppose à Eumèle, héros apollinien. Plus tard, après les événements narrés par l'Iliade, il sera tué par Memnon, fils de l'Aurore ${ }^{33}$.

Une autre contestation s'élève alors: Ménélas accuse Antiloque d'avoir gagné par traîtrise, en un mot d'avoir triché. Du coup, et pour éviter une querelle, Antiloque propose de donner le prix à Ménélas. Sa réaction est habile, il désarme ainsi la colère de l'Atride, qui, après une courte leçon de morale, se contentera du troisième prix. Protégé de Poseidon, Antiloque garde donc le second prix, une cavale; les chevaux sont proches de Poseidon, mais c'est par la ruse et l'intelligence qu'Antiloque a obtenu le second prix.

En somme, la course de chars se termine par une double victoire d'Athéna : de son protégé Diomède sur Eumèle aidé par Apollon et d'Antiloque sur Ménélas, personnage plus puissant et qui est proche d'Arès.

Les autres épreuves sont beaucoup moins importantes, leur description est brève et les prix obtenus sont plus modestes. Le pugilat oppose Épéios, fils de

\footnotetext{
29 Antiloque parle à ses chevaux comme s'il s'adressait à des êtres humains. Hector fait la même chose quand il excite ses chevaux ( $\Theta$ 185-197).

30 Sur les recours d'Antiloque à la ruse et son lien avec Athéna, cf. P. WATHELET, Dict. des Troyens, op, cit. (n. 1), p. 145-146, $\mathrm{n}^{\circ} 3^{\mathrm{N}} \mathrm{A} \beta \lambda$ poos.

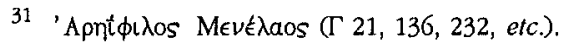

32 Sur Mérion, cf. P. WATHELET, Dict. des Troyens, op. cit. (n. 1), p. 168-170, nº 9 'A śápas.

33 La mort d'Antiloque est rappelée à plusieurs reprises dans l'Odyssée, qui précise en $\delta 188$ qu'il a été tué par le fils de l'Aurore. L'événement était raconté dans l'Êthiopide (Résumé de Proklos, 188-190 Severyns).
} 
Panopeus, et Euryalos, fils de Mékisteus. Le premier lance une provocation qui semble quelque peu présomptueuse, mais l'Odyssée $(\theta 493)$ rappelle le fameux cheval de bois qu'Épeios fit avec Athéna. Épeios est donc un artisan fameux qui, en tant que tel, est proche de la déesse. Euryalos s'appelle «LargeMer ${ }^{34}$, il est fils de Mékisteus, «le très long ${ }^{35}$, qui pourrait porter un nom de géant ou désigner au moins un être monstrueux. Il n'est pas impossible qu'Euryalos soit proche de Poseidon ${ }^{36}$. On aurait donc dans le pugilat une opposition entre Athéna (Épeios) et Poseidon (Euryalos), opposition qui se termine par la victoire d'Athéna, comme chaque fois que les deux divinités s'opposent.

La lutte opposera des champions de plus haut rang : Ajax, fils de Télamon, et Ulysse. Comme aucun des deux n'arrive à abattre l'autre, Ulysse frappe Ajax au jarret, par derrière. Il le fait tomber en arrière, mais sans succès. Achille interrompt le combat et donne à tous deux des prix égaux.

$\mathrm{Au}$ moment où le combat est interrompu, Ulysse était en train de prendre l'avantage par une ruse - le héros est fidèle à lui-même. L'auditoire d'Homère sait que la contestation reprendra ${ }^{37}$.

L'épreuve qui suit est la course à pied. Elle oppose Ulysse - décidément infatigable -, Ajax, fils d'Oilleus, et Antiloque. Alors qu'Ajax est sur le point d'emporter l'épreuve, Ulysse invoque Athéna et celle-ci fait glisser Ajax dans une bouse de vache. Il tombe et c'est le nez et la bouche pleins de bouse qu'il vient chercher son deuxième prix : une vache ${ }^{38}$ ! Homère aime se moquer du fils d'Oíleus. Antiloque est le troisième, mais là aussi la ruse est payante, car il met tant de bonne grâce à prendre le troisième prix qu'Achille y ajoute un demi-talent d'or.

Le combat opposera Ajax, fils de Télamon, et Diomède. Les deux héros s'affrontent. Les Achéens, " pris de peur pour Ajax» ( $\Psi$ 822), mettent fin à l'affrontement et proposent de leur donner une part égale du prix. Mais c'est à Diomède qu'Achille remet le grand poignard ( $\Psi$ 824). Encore une fois, le protégé d'Athéna est favorisé.

Les trois dernières épreuves intéressent moins notre propos, elles sont brièvement racontées en soixante-seize vers. Le lancer du disque oppose Polypoites, Léonteus, Ajax, fils de Télamon, et Épeios. C'est le premier qui l'emporte. Fils de Peirithoos, il incarne la résistance de la civilisation contre la

34 H. von KAMPTZ, Homerische Personennamen. Sprachwissenschafliche und bistorische Klassifikation, Göttingen, Vandenhoeck-Ruprecht, 1958, p. 28, §10 a 3; p. 71, §22 a 2; p. 194, \$66.

35 H. von KAMPTZ, tbid., p. 122, \$42 a 1; p. 124, \$42 c 1; p. 228, §67; p. 294, \$80 a.

36 Euryalé est notamment le nom d'une Gorgone et celui d'une fille de Minos qui fut mère d'Orion par les œuvres de Poseidon IPs.-HÉsIOdE, fgt 148 (a) M.-W.; PHÉrÉcyde (3 F 52 Jacoby) et Ps.-APOLL., $B .$, I, 4, 3]. Il existe d'autres versions de la naissance d'Orion.

37 Ce sera à l'occasion de l'attribution des armes d'Achille après la mort du héros. Cf. $\lambda$ 543564 et Étbiopide (Résumé de Proclos, 202-203 Severyns).

38 A. SEVERyNS, Homère, III. L'artiste, Bruxelles, Office de Publicité, 1948 (Collection lebègue), p. 125. 
sauvagerie incarnée par les Centaures. Le tir à l'arc est disputé par Teucros et Mérion. On est ici dans le domaine d'Apollon, le dieu archer, et, comme Teucros n'a pas invoqué le dieu et que Mérion l'a fait, c'est ce dernier qui remporte le prix. Au lancé de la javeline, Agamemnon et Mérion se présentent, mais, pour éviter un impair et sans que l'épreuve ait lieu, Achille donne le premier prix à Agamemnon. Ce dernier n'a pris part à aucune des autres épreuves et le prix qu'il reçoit sans concourir ne fait que confirmer la faible estime que l'on a pour son mérite personnel.

Ainsi, dans l'Iliade, Athéna apparaît comme une divinité essentielle à l'action, et partout présente. Dans l'ensemble, l'Odyssée fait le même portrait que l'Iliade, mais la déesse y occupe une place encore plus importante. Elle patronne le travail des artisans. Comme on l'a signalé plus haut, elle a aidé Épeios qui avait construit le cheval de bois ( $\theta$ 493); avec Héphaistos, elle a instruit un artiste habile à nieller, or sur argent, un chef-d'œuvre, ainsi qu'il est dit dans une comparaison $(\zeta 232-234=\psi 159-161)$. Elle dirige aussi les travaux féminins; elle avait accordé l'adresse des mains aux tisseuses de Phéacie ( $\eta$ 110). Comme Pénélope fait une prière à Artémis, elle évoque les filles de Pandareos, auxquelles Athéna avait donné l'art des beaux ouvrages ( $v$ 72). Parlant de la tapisserie de Pénélope, Antinoos rappelle que la reine s'est fiée aux travaux d'Athéna et à son art de la ruse $(\beta \text { 116-117) })^{39}$. Athéna est une divinité poliade, son culte est particulièrement fameux à Athènes. En quittant Schérie, elle part pour Marathon, Athènes et le foyer d'Érechthée ( $\eta$ 79). Au pays des Phéaciens, à l'entrée de la ville se trouve un sanctuaire d'Athéna dans un bois de peupliers ( $\zeta$ 291-292 $)^{40}$. C'est là qu'Ulysse s'arrêtera, tandis que Nausikaa et ses compagnes poursuivront leur chemin.

L'Odyssée rappelle qu'Athéna n'a cessé de combattre les Troyens et de protéger les Achéens, aussi longtemps qu'a duré le siège de la ville de Priam. Ainsi, taquinant Hélène, Ménélas raconte à Télémaque que cette dernière est venue, à Troie, tourner autour du cheval de bois en imitant les voix des femmes des héros achéens enfermés à l'intérieur. Seul Ulysse a pu les empêcher de se trahir jusqu'au moment où Athéna a emmené Hélène ( 8 289). Lors de la prise de Troie, les choses se sont gâtées à cause de l'attentat qu'Ajax le Locrien a perpétré sur Cassandre. J'ai eu l'occasion de souligner combien Cassandre était proche d'Athéna ${ }^{41}$ et la violence d'Ajax contre elle dans le temple même de la déesse ne pouvait qu'irriter doublement celle-ci $(\gamma$ 130-

39 Le personnage de Pénélope est étrange et à bien des égards mystérieux. Le jour, elle se livre à une besogne typiquement féminine, la tapisserie, mais, la nuit, elle défait son travail, ce qui représente une activité masculine. - Cf. M. ELIADE, Naissances mystiques. Essat sur quelques types d'initiation, Paris, Gallimard, $1959^{4}$, p. 101, et plus récemment I. PAPADOPOUOU-BELMEHDI, Le Chant de Pénélope, Paris, Belin, 1994 (L'Antiqutié au présent).

40 Curieusement, ce sanctuaire d'Athéna est à l'entrée de la ville, alors que Poseidon en a un au centre de celle-ci. C'est une situation opposée à celle qui est habituelle. Le monde des Phéaciens est un monde qui prend, sur plusieurs points, le contre-pied du monde réel.

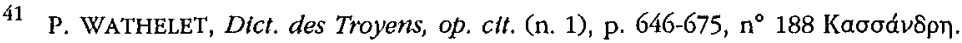


$152-\delta$ 498-503). Le fait le plus remarquable concernant le rôle d'Athéna dans l'Odyssée est l'aide efficace qu'elle apporte à Ulysse et aux membres de sa famille.

Pour se manifester, elle prend de nombreux déguisements. Dans la Télémachie, elle revêt successivement la forme de Mentès ${ }^{42}$ ( $\alpha$ 96-323), puis, beaucoup plus longuement, celle de $\operatorname{Mentor}^{43}(\beta 268-\gamma 372)$. Sous la première forme, elle s'indigne de l'attitude des prétendants et conseille à Télémaque de se rendre à Pylos et à Sparte, avant de revenir à Ithaque pour se débarrasser d'eux (a 253-305). Déguisée en Mentor, Athéna donne ses instructions à Télémaque, puis elle endort les prétendants et appelle Télémaque. Le vaisseau part d'autant plus facilement que la déesse envoie un zéphyr qui le pousse. À l'arrivée à Pylos, Athéna/Mentor ne cesse d'aider Télémaque, qui, sinon, se trouverait très embarrassé. Comme Nestor, intarissable bavard, se perd en discours, Mentor l'engage fermement à terminer le sacrifice commencé ( $\gamma$ 331336). Nestor invite Mentor et Télémaque à loger chez lui. Mentor accepte pour Télémaque, en lui prodiguant ses derniers conseils : lui-même retournera au vaisseau, afin de partir dès le matin ( $\gamma$ 357-370). Dans les deux passages, Athéna disparaît en changeant de forme à la vue de tous : dans le premier cas, elle se transforme en " oiseau de mer" ( $\alpha$ 320), dans le second, en orfraie ( $\gamma$

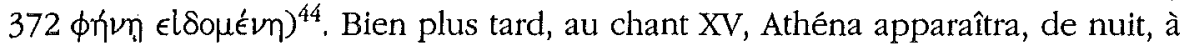
Télémaque, qui a une insomnie. Elle le presse de rentrer au plus vite à Ithaque, pour sauver son patrimoine. Elle lui donne des indications afin de déjouer l'embuscade des prétendants. L'aide d'Athéna à Télémaque se manifeste donc jusqu'à Pylos. Pour le reste du voyage, la déesse laisse le fils d'Ulysse aux mains du Nestoride Pisistrate, elle n'interviendra qu'une seule fois, à la faveur de la nuit, pour le faire rentrer à Ithaque ${ }^{45}$.

Comme on l'a vu, vis-à-vis de Pénélope, les interventions de la déesse sont plus discrètes: Antinoos reproche à Pénélope de s'être fondée sur les dons d'Athéna, afin de tromper les prétendants avec sa tapisserie. Aux moments décisifs, Athéna fait naître chez Pénélope le désir d'apparaître aux prétendants ( $\sigma$ 158). Pour le reste, la déesse se borne à calmer la peine ou l'inquiétude de la femme d'Ulysse en octroyant à quatre reprises à celle-ci « le plus doux des sommeils » $(\alpha 363-364=\mathrm{T} 603-604, \phi 357-358$ et $\sigma$ 188). Au chant XVI, la déesse profite du sommeil de Pénélope pour lui envoyer l'image $(\epsilon l \delta \omega \lambda \circ \nu)$ de sa soeur Iphthimé, qui la rassure sur le sort de Télémaque ( $\delta$ 795-841). Au chant

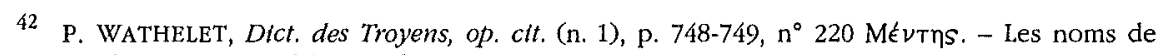
Mentès et de Mentor sont bâtis sur la même racine.

43 P. WATHELET, Mentor/Athéna, maître d'initiation dans lOdyssée, in Uranle, 4 (1994), p. 11-23.

44 L. BODSON, 'IEPA ZRIA, Contribution à l'étude de la place de l'animal dans la religion grecque ancienne, Bruxelles, Académie, 1978 (Mém. de l'Acad. de Belg., Classe des Lettres, 68, 2), p. 96.

45 Sur la chronologie de l'Odyssée et les problèmes délicats qu'elie pose, cf. E. DELEBECQUE, Construction de lOdyssée, Paris, Les Belles Lettres, 1980. 
XVIII, la déesse se sert du sommeil de Pénélope pour la farder avant qu'elle n'apparaisse à Ulysse ( $\sigma$ 187-195).

Athéna est avant tout la protectrice d'Ulysse ${ }^{46}$ lui-même. Déjà, à Troie, elle a favorisé son protégé, ainsi que Nestor le rappelle à Télémaque $(\gamma 218)$. La déesse intervient en faveur du héros auprès de Zeus et des autres dieux de l'Olympe ( $\alpha$ 44-95, $\in$ 5-27). Au moment où, sous la tempête levée par Poseidon, Ulysse est en difficulté, Athéna profite de l'inattention de Poseidon pour calmer les flots et amener le héros chez les Phéaciens ( $\epsilon$ 380-387). Quand, enfin, il aborde, elle l'endort ( $\epsilon$ 491). Depuis l'arrivée d'Ulysse chez les Phéaciens jusqu'à la fin de l'Odyssée, l'aide de la déesse est constante et vigilante. C'est un jeu subtil de déguisements successifs d'Athéna elle-même. Elle prend la forme d'une amie de Nausikaa pour apparaître à celle-ci au cours d'un songe ( $\zeta$ 112). Alors qu'Ulysse marche vers le palais d'Alkinoos, Athéna se présente à lui sous la forme d'une petite fille, «une cruche à la main ». Elle le met au courant de ce qu'il doit savoir. Ayant transmis son message, la déesse disparaît vers la mer, Marathon et Athènes ( $\eta$ 81). Au début du chant VIII, Athéna, de nouveau déguisée - elle a pris la forme du héraut d'Alkinoos - s'en va en ville ameuter les Phéaciens pour les inciter à venir voir Ulysse. Plus loin, lors du concours de jet du disque, Athéna prend les traits d'un homme pour souligner que le disque d'Ulysse est allé plus loin que celui de tous les autres $(\theta$ 195-197). Alors que les Phéaciens ont ramené Ulysse endormi à Ithaque et qu'il se réveille, Athéna lui apparaît sous les traits d'un jeune berger adolescent $(v$ 185-249). Comme Ulysse lui raconte une histoire qu'il invente, Athéna reprend des traits féminins pour se donner à connaître au héros, puis elle l'aide à dissimuler les richesses que les Phéaciens lui ont données ( $\nu$ 256-371). Plus tard, quand Ulysse et Télémaque seront réunis dans la cabane d'Eumée, Athéna se présente aux yeux du seul Ulysse sous la forme d'une grande et belle femme, artiste en beaux ouvrages, elle est invisible à Télémaque, mais identifiée par les chiens ( $\pi$ 154-176). Toujours inconnu, Ulysse se couche dans l'avant-pièce de son propre palais, il voit les servantes qui rejoignent les prétendants et il s'en indigne vivement. Il médite sa vengeance. Athéna se montre à lui sous les traits d'une femme ( $v$ 31). Au moment où Ulysse et Télémaque massacrent les prétendants, Athéna leur apparaît en Mentor ( $\chi 205$ 207), Ulysse, qui a reconnu la déesse, l'invoque, tandis qu'un des prétendants menace Mentor, provoquant une réaction violente d'Athéna, qui excite Ulysse au courage. Ensuite la déesse se change en hirondelle, elle se pose sur les poutres du faîte $(\chi 240)$. Médon signale aux habitants d'Ithaque, que, sous l'apparence de Mentor, Athéna se tenait auprès d'Ulysse pour l'aider dans sa lutte contre les prétendants ( $\omega$ 443). Au moment où les parents des prétendants vont se déchaîner contre Ulysse, Athéna se montre sous le déguisement de Mentor ( $\omega$ 502), elle excite Laërte.

46 M. MÜLLER, Athene als Götlicbe Helferin in der Odyssee. Untersucbungen zur Form der episcben Artstie, Heidelberg, Winter, 1966. 
Non seulement Athéna prend des formes diverses, mais elle change l'apparence des personnages qu'elle protège. Accompagné de deux chiens, Télémaque arrive à l'assemblée d'Ithaque, « Athéna le parait d'une grâce céleste » ( $\beta$ 12). Quand Ulysse se présente à Nausikaa après s'être lavé et habillé, Athéna le fait apparaître plus grand et plus fort, « elle déroulait de son front des boucles de cheveux aux reflets d'hyacinthe »... «Athéna versait la grâce sur la tête et le buste d'Ulysse » ( $\zeta$ 231-235). Le héros se met en route vers le palais d'Alkinoos, Athéna l'enveloppe d'une nuée épaisse, afin qu'aucun des Phéaciens ne puisse le reconnaître ( $\eta 15$ ). La nuée ne se dissipera que quand, arrivé dans le palais d'Alkinoos, il se jette aux pieds d'Arété ( $\eta$ 140). Alors qu'Ulysse a été reçu chez Alkinoos, Athéna incite les Phéaciens à venir voir Ulysse qu'elle pare d'une grâce extraordinaire ( $\theta$ 19). Ramené à Ithaque par les Phéaciens, Ulysse s'éveille de son sommeil, Athéna a versé autour de lui une nuée "afin que, de ces lieux, il ne reconnût rien et qu'il apprît tout d'elle » ( $v$ 190). Ulysse et Télémaque sont ensemble dans la cabane d'Eumée, mais Télémaque ignore qu'il est en présence de son père. Athéna apparaît au seul Ulysse, le fait sortir et le convainc de se donner à connaître ( $\pi$ 155-162). Elle touche Ulysse de sa baguette d'or, et lui rend l'apparence de la jeunesse ( $\pi$ 172-176). Plus tard, quand Eumée revient, Pallas touche à nouveau Ulysse, pour lui rendre sa forme de vieillard loqueteux ( $\pi$ 456-457). Rentré à Ithaque, Télémaque rencontre Pénélope, puis il apparaît aux prétendants, Athéna le parait d'une grâce céleste ( $\rho$ 63). Quand il a massacré les prétendants, Ulysse se baigne avant de se donner à connaître à Pénélope. Sur sa tête, Athéna répandait la beauté $(\psi 156)$. Ulysse et Télémaque se rendent chez Laërte, Athéna les couvre d'un voile de nuit pour qu'ils passent inaperçus ( $\psi 371)$. Laërte reçoit Ulysse et Télémaque, il se prépare au repas, prend un bain, Athéna le rendait plus grand et plus fort que jadis ( $\omega$ 367-369).

Ainsi, Athéna ne cesse d'apporter son aide à Ulysse et aux siens. La déesse pousse même la rouerie jusqu'à susciter par deux fois les moqueries et les insultes des prétendants envers Ulysse, afin d'exciter la colère du héros à leur endroit ( $\sigma$ 346, $v$ 284).

Deux questions doivent être évoquées ici : pourquoi Athéna n'intervientelle en faveur d'Ulysse que dans une partie de l'Odyssée seulement ? Et pourquoi la déesse multiplie-t-elle les déguisements?

Alors qu'Athéna ne cesse de protéger Ulysse de la manière la plus directe dès le moment où le héros, naufragé, est sur le point d'aborder chez les Phéaciens, elle est étonnamment absente pendant tout le périple du fils de Laërte. Depuis le passage d'Ulysse et de ses compagnons chez les Cicones jusqu'au moment où, lassé de l'amour de Calypso, il souhaite rentrer à Ithaque, Athéna ne se manifeste pas. Et pourtant Ulysse connaît un nombre considérable de dangers où l'aide de la déesse lui aurait été bien utile. Il l'invoque même alors qu'il est dans la grotte de Polyphème ( $(317)$, mais sans effet. La première intervention de la déesse se produira à la première assemblée des dieux où elle obtient d'aller trouver Télémaque ( $a$ 44-62). Lors de la seconde assemblée, elle 
fera en sorte qu'Hermès soit envoyé à Calypso pour lui intimer l'ordre de laisser partir Ulysse $(\epsilon$ 7-20).

Le poète a senti qu'il y avait là un problème et, lorsqu'Athéna et Ulysse se retrouvent ensemble à Ithaque au moment où les Phéaciens viennent de le ramener, Ulysse remarque que le dévouement de la déesse à son égard a cessé depuis la fin de la guerre de Troie jusqu'à son arrivée chez les Phéaciens. Il précise qu'il ne l'a pas « sentie embarquée à son bord pour lui épargner des maux » ( $\nu$ 319). La déesse excuse son absence par un prétexte : elle n'a pas voulu combattre Poseidon, le frère de son père, qui avait contre Ulysse tant de rancune, après qu'il eut aveuglé son fils ( $\nu$ 341-343). L'excuse est bien peu satisfaisante : il s'est écoulé du temps entre la fin de la guerre de Troie et l'aveuglement de Polyphème et, dès l'arrivée d'Ulysse chez les Phéaciens, Athéna a cessé de redouter la colère de Poseidon, qui était pourtant loin d'être apaisée.

D'autres raisons ont été invoquées, dont la "colère d'Athéna » 47 . S'adressant à Calypso, Hermès lui dit qu'un héros est près d'elle « le plus lamentable de tous ceux qui, sous la grand'ville de Priam, étaient allés combattre neuf ans et, le dixième, ayant pillé la ville, rentrèrent au logis; Athéna, qu'ils avaient offensée au départ, déchaîna la tempête et des vagues énormes; son équipage entier de braves succomba; mais la houle et le vent sur ces bords le jetèrent » ( $\epsilon$ 106-111 - trad. V. Bérard). Apparemment la colère d'Athéna visait surtout Ajax, fils d'Oilée à la suite de son attentat contre Cassandre, et secondairement les Achéens qui ne l'avaient pas assez puni ( $\delta$ 499-510 ${ }^{48}$. Ici encore, si telle était la bonne explication, on ne voit pas pourquoi Athéna aurait subitement mis fin à sa colère. À l'inverse d'Apollon dont les colères sont brutales, mais passagères, celles d'Athéna sont durables et persévérantes. Un autre argument peut être invoqué qui explique la longue abstention de la déesse. En fait, dès qu'Ulysse quitte le pays des Cicones et qu'avec ses compagnons il arrive chez les Lotophages, il pénètre dans un domaine qui n'est plus celui de la réalité, mais un monde de féerie et de monstres. À bien des égards, c'est l'Autre Monde ${ }^{49}$. Or les dieux olympiens répugnent à s'aventurer dans le domaine souterrain. Lorsqu'Ulysse arrive chez Circé et qu'il se trouve en grand danger d'être victime de l'enchanteresse, Hermès, le dieu psychopompe, se présente à lui pour lui donner un antidote, le fameux $\mu a \hat{\lambda} \nu$ (a 275-308). C'est le même Hermès qui sera envoyé chez Calypso, comme il l'avait été dans le royaume d'Hadès pour en ramener Corè. Athéna ne reprendra son rôle de protectrice d'Ulysse que quand celui-ci revient

\footnotetext{
47 J. Strauss CLAY, The Wrath of Athena. Gods and Men in the Odyssey, Princeton Univ. Press, 1983.

48 Le thème sera repris dans le Prologue des Troyennes d'Euripide (v. 48-94).

49 On rapprochera, par exemple, le $\Lambda \pi j \theta \eta$ où les âmes des morts perdaient le souvenir de leur vie passée et les Lotophages, mangeurs de Lotos, qui perdent la mémoire.
} 
vers le monde de la réalité, c'est-à-dire chez les Phéaciens, dont le royaume se trouve à la frontière des deux domaines ${ }^{50}$.

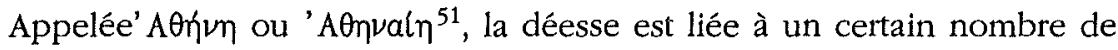

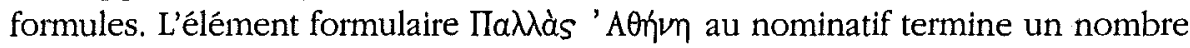
considérable de vers, tant dans l'Iliade que dans l'Odyssée. Le nom de Pallas reste malheureusement des plus obscurs ${ }^{52}$. L'élément connaît quelques

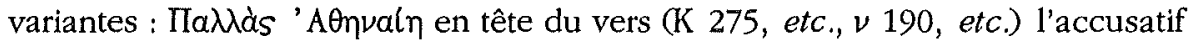

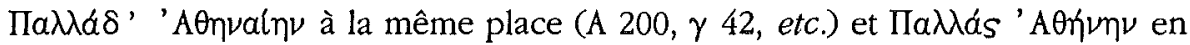
fin de vers (E 510).

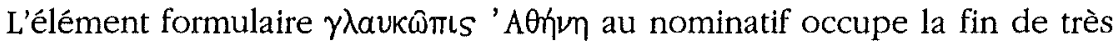
nombreux vers de l'Iliade (B 172, etc.) et de l'Odyssée (a 319, etc.). On trouve

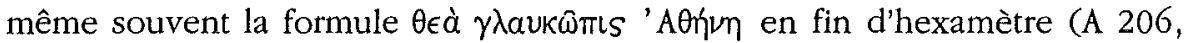
etc.; a 44, etc.), avec $\theta \epsilon a ́$, féminin de $\theta \epsilon o ́ s$ qui est un éolisme ${ }^{53}$, mais $\theta \epsilon a ́$ pourrait s'être substitué à $\theta \epsilon \delta s$, sans entraîner d'altération métrique. $\Gamma \lambda \alpha u k \omega \pi \mathrm{m} s$ est une épithète propre à Athéna, dont le sens originel devait être «à la face " ou «aux yeux de chouette», même si le composé a pu être compris comme "aux yeux étincelants, terribles ${ }^{54}$. Ici encore, l'élément formulaire est

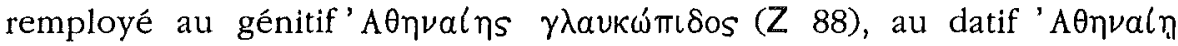
$\gamma \lambda a u k \omega ́ m \delta$ (I 390 , etc.), chaque fois au milieu du vers et, à l'accusatif,

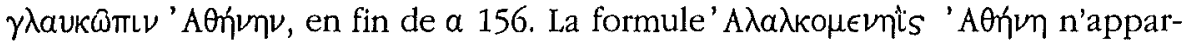
aît qu'une seule fois dans un vers répété $(\Delta 8=\mathrm{E} 908)$. L'épithète

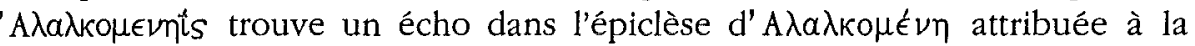

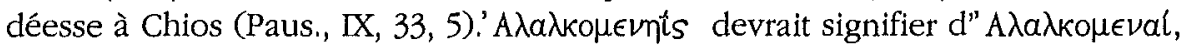
ville de Béotie, dont le nom est dérivé de $\alpha \lambda a \lambda \kappa \in \hat{\imath} \nu$ " repousser un danger », mais Aristarque comprenait'A $\lambda a \lambda \kappa o \mu \epsilon \nu \eta$ is, comme «protectrice $»^{55}$. Le lien de l'épithète avec le toponyme est d'autant plus probable que le premier hémistiche de $\Delta 8=\mathrm{E} 908$ mentionne "H $\tau^{\tau}$ " $A \rho \gamma \epsilon l \eta$, et il semble bien que la déesse avait un sanctuaire important à 'A $\lambda a \lambda \kappa o \mu \epsilon \nu a l$. Néanmoins, même si elle

50 Les Phéaciens sont voisins, à l'origine, des Cyclopes ( $\zeta 5$ ). Comme Polyphème, ils descendent de Poseidon ( $\eta$ 56) et sont donc attachés à l'origine aux régions marginales en bordure du monde civilisé où se retrouvent tous les monstres, ce qui explique leur caractère inquiétant et surtout la prudence d'Ulysse à leur égard. Homère prend soin d'indiquer qu'ils ont quitté leur résidence originelle pour se rapprocher du monde des humains $(\zeta 6-7)$. Ce sont des passeurs $(v$ 174) qui se tiennent entre le monde de la féerie et celui des humains.

51 P. Chantraine, Dict. ét., p. 27-28, s.v.'Agrinn. - La question se pose de savoir si Athéna est la déesse d'Athènes (ce que ferait croire la seule mention qui apparaisse de la déesse sur les tablettes en linéaire $\mathrm{B}$, à Cnossos, sous la forme A-ta-na-po-ti-ni-ja-cf. $\mathrm{n}$. 75) comme le croit W. BURKERT (Greek Religion, Harvard, 1985, p. 139), qui note que le suffixe $\eta \nu \eta$ est un suffixe de noms de ville, ou si Athènes est la ville d'Athéna, comme le pense Pierre Chantraine.

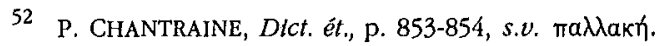

53 P. WATHELET, Les tratts éollens dans la langue de l'épopée grecque, Rome, Ateneo, 1970, p. 354-355.

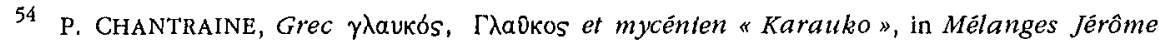
Carcopino, Paris, Hachette, 1966, p. 193-203.

55 P. ChanTRAINE, Dict. ét., p. 57, s.v. a $\lambda \epsilon \xi \omega$. 
est secondaire au point de vue strictement étymologique, l'interprétation d'Aristarque ne peut être écartée, d'autant plus qu'elle se trouve renforcée par

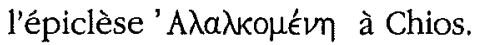

Athéna est encore qualifiée de $\dot{\epsilon} \pi l \rho p o \theta 0 s$ « qui secourt ${ }^{56}$, mais dans un seul vers $(\Delta 390)$. L'épithète apparaît dans d'autres contextes. Elle est aussi $\pi \circ \lambda$ úßoùos « riche en conseils » en $E 260$ et $\pi$ 282, mais les deux vers

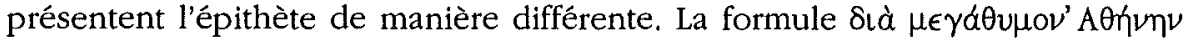
termine $\theta 520$ et $\nu 121$, l'adjectif $\mu \epsilon \gamma a ́ \theta v \mu o s$ est fréquent et appliqué à des personnages très divers.

Le nom de la déesse est uni à celui de Poseidon en fin des vers $\Upsilon 115$ et $\Phi$

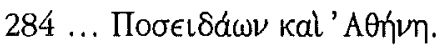

Appelée cette fois 'A $\theta \eta v a i \eta$, « l'Athénienne ${ }^{57}$, la déesse apparaît dans

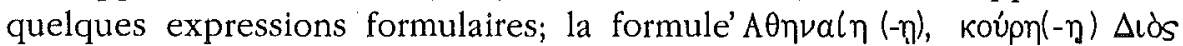
alyı́x Xoto termine les vers $\mathrm{E} 733=\Theta 384 ; \nu 252(=371, \omega 547) ; \delta 752 ; \gamma 42 ; \omega$

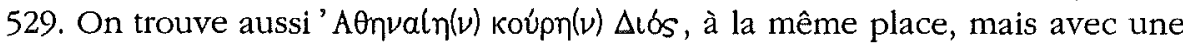

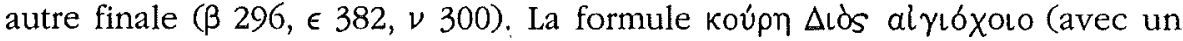
génitif thématique en -oto qui caractérise l'achéen et le thessalien) est liée à 'Aonval $\eta$, mais, à date plus ancienne, elle aurait pu être employée dans un autre contexte.

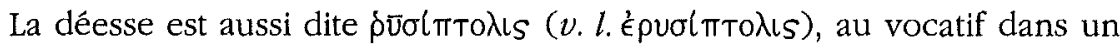

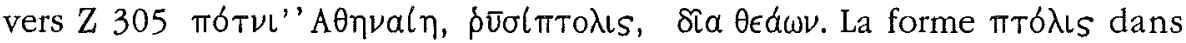
$\delta \overline{v \sigma l \pi T o \lambda}$ s est un trait achéen ou thessalien ${ }^{58} ; \theta \in \alpha \omega \nu$ constitue un éolisme archaïque qui ne peut recouvrir un plus ancien $\theta \in \hat{\omega} \nu \nu^{59}$.

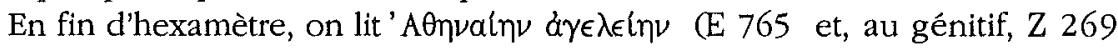
$=279$, etc., Hés., Théog., 318). L'épithète, «qui emmène du butin », vient de ${ }^{*} \alpha \dot{\alpha} \gamma \epsilon-\lambda \bar{\alpha} \mathrm{F}\left\llcorner a \nu>{ }^{*} \alpha \gamma \epsilon \lambda \eta\llcorner\alpha \nu>\alpha \gamma \epsilon \lambda \epsilon l \eta \nu\right.$, cette dernière forme ne peut recouvrir la forme originelle ${ }^{60}$. Elle semble bien ionienne, ce qui en ferait un trait très récent dans la tradition épique.

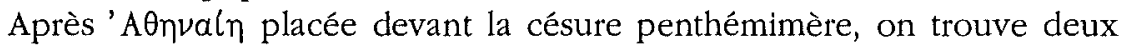

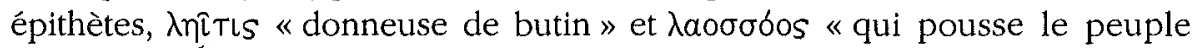

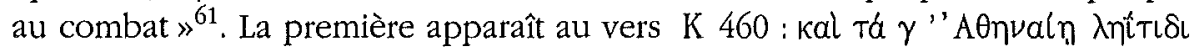

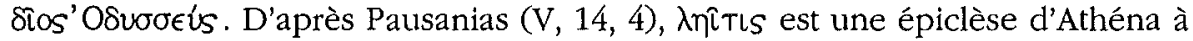

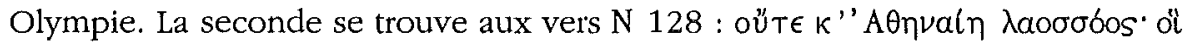

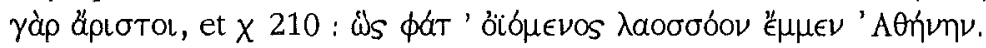

P. ChantRaine, Dict. ét., p. 977, s.v. pobos.

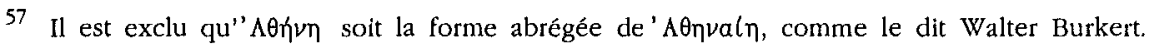

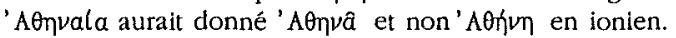

58 P. WATHELET, Les traits éoliens, op. clt. (n. 53), p. 92-95.

59 Cf. n. 53.

60 P. Chantraine, Dict. ét., p. 626, s.v. $\lambda \epsilon i a$.

61 P. ChanTraine, Dict. ét., p. 997, s.v. oєúoual. 
Au nominatif ou à l'accusatif, l'adjectif occupe la même position dans toute une série de vers (P 398; Ү 48; 79; o 244; $\chi 210 ;$ Bouclier, 37) et il est appliqué à d'autres divinités (Arès $\mathrm{P} 398$, Éris $\Upsilon 48$, Apollon $\Upsilon 79$ ).

Athéna est liée à Héra en fin de $\Delta 20=\Theta$ 457; $E$ 418; $\Theta$ 444; 447; I 254;

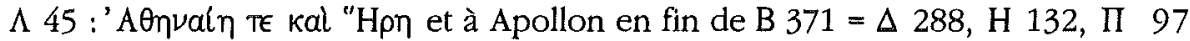

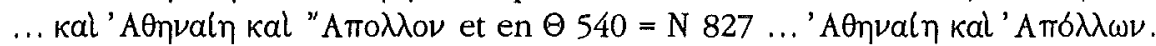

Les formules les plus fréquentes semblent être celles qui contiennent le

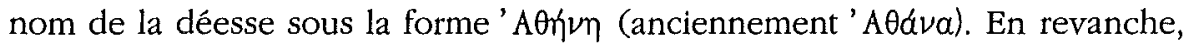

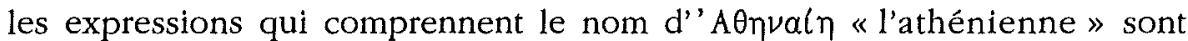
moins souvent utilisées et d'apparence moins traditionnelles. Certaines des épithètes font allusion à l'activité strictement guerrière de la déesse, elles paraissent plutôt liées à la forme 'AOnvaln du théonyme. Le développement de

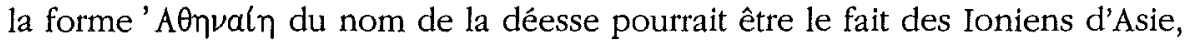
qui ont pu se sentir proches des Ioniens occidentaux et d'Athènes, mais c'est là une hypothèse indémontrable.

Athéna est apparemment à l'origine la déesse qui veille sur la maison, sur le palais ${ }^{62}$. Comme Hestia, la déesse du foyer ${ }^{63}$, elle est vierge : leur mission leur interdit toute autre activité. Comme Hestia également, elle est peu mêlée à des récits mythiques, du moins au début ${ }^{64}$. La maison est par nature liée à la terre et la déesse présente des aspects chthoniens que l'on retrouve d'ailleurs auprès de la majorité des déesses grecques. Le serpent qui l'accompagne dans certaines de ses représentations, et dont la plus célèbre est la statue chryséléphantine du Parthénon a très probablement une valeur apotropaïque ${ }^{65}$ : il écarte les indésirables de la maison ou du palais. La garde qu'assure la déesse est spécialement vigilante la nuit, ses yeux sont comme ceux de la chouette qui percent les ténèbres : elle est $\gamma \lambda \alpha u \kappa \omega \hat{\pi} \iota^{66}$. Le côté chthonien d'Athéna la met en relation avec Poseidon, celui qui porte la terre ou qui l'ébranle. Comme souvent dans le mythe, la relation est faite tantôt de bonne entente, tantôt d'opposition. C'est ce dernier côté qui apparaît dans l'Odyssée.

Sa qualité de protectrice de la maison, du palais et par conséquent de la ville, se marque par le rôle joué, à Troie et ailleurs, par le Palladion, statue d'Athéna, qui constitue une sorte de talisman pour la cité.

62 M.P. NILSSON, The Minoan-Mycenaean Religion and its Survival in Greek Reltgion, Lund, Gleerup, $1950^{2}$.

63 Cf. Hymne ps.-bom. à Apbrodite, 21-32.

64 On reste surpris de voir le rôle à tout le moins discret d'Athéna dans les mythes attiques, même si on ne tient pas compte du mythe de Thésée qui est probablement plus lié à Trézène qu'à Athènes (cf. F. JOUAN, Les légendes attiques dans le théatre de Sophocle, in Estudios de Mitologia griega I: Mitos en la Literatura Griega arcaica y clástca (V Coloquio internacional de Filología griega, Madrid, 2-5 de Marzo de 1994), à paraître.

65 L. BODSON, 'IEPA ZOIA, op.ctt. (n. 44), p. 77-79.

66 L. BODSON, 'IEPA ZOIA, op. cit. (n. 44), p. 97. 
Homère ne dit rien de la naissance d'Athéna, la version de cette naissance que donne Hésiode ${ }^{67}$ et qui est largement reprise dans l'iconographie ${ }^{68}$, preuve du fait qu'elle est largement répandue dans le monde grec, explique qu'Homère n'en ait pas parlé : l'auteur de l'Iliade passe volontiers sous silence les faits trop marqués par le merveilleux. Une tradition tardive fait d'Athéna la fille de Pallas qu'elle aurait tué pour pouvoir s'approprier sa force ${ }^{69}$. Walter Burkert $^{70}$ rapproche les deux versions : dans les deux cas, la naissance de la déesse impose que son père soit frappé, «détruit», et elle hérite de toute sa force. Déesse parce que liée à la terre, comme la maison ou la ville, Athéna a hérité de son père une fonction de défense et de protection ${ }^{71}$.

Protectrice de la maison, Athéna est naturellement proche d'Héra, déesse qui incarne les institutions, spécialement celle du mariage ${ }^{72}$ et qui possédait en Grèce les temples, les «maisons » les plus anciennes ${ }^{73}$. Ainsi, l'alliance presque constante qui apparaît entre les deux déesses dans l'Iliade pourrait-elle ne pas être de circonstance. Athéna exécute les ordres d'Héra, comme la gardienne du logis est soumise à la maîtresse de maison.

Mais déjà dans l'Iliade, Athéna prend seule des initiatives. La tendance sera encore plus marquée dans l'Odyssée. Il est des stratèges pour qui la meilleure défense réside dans l'attaque. C'est ce qui a dû arriver à Athéna : son intelligence active, sa ruse l'ont sans doute amenée à sortir d'un rôle purement défensif. Les guerriers chargés de défendre la ville en sont sortis pour se lancer dans des expéditions à l'extérieur et Athéna les a accompagnés, elle a continué de les protéger. Son intelligence pratique l'a aussi amenée à les aider à se tirer des moments les plus difficiles et cette aide n'est pas seulement guerrière, mais aussi appliquée à d'autres domaines. Comme Ulysse et Télémaque le soulignent dans l'Odyssée, Ithaque n'a rien d'une île naturellement prospère ${ }^{74}$. Ulysse compense sa fortune modeste par un surcroît d'intelligence et c'est ainsi qu'il s'impose comme un des premiers parmi les princes achéens, alors que

\footnotetext{
67 Théog., 886-900 et 924-929 (voir toutefois E 880).

68 P. DEMARGNE, art. Athena, in LIMC, II (1984), p. 955-1044.

69 Schol. Lycophr, 355; CIC., De Nat. Deorum, III, 59.

70 W. BURKERT, op. cit. (n. 51), p. 139-143 et notes.

71 W. BURKERT, ibid., a certainement raison de lier la virginité de la déesse au fait qu'elle soit née de son seul père.

72 W. BURKERT, ibid., p. 131-135.

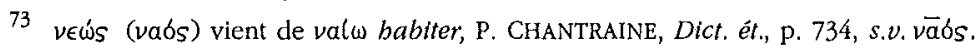

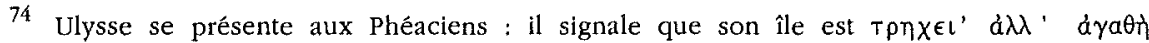
коupotpobos ( 27 ). En quelques mots, Ulysse énonce bien le problème démographique de la Grèce du temps d'Homère, peu de fertilité et une population nombreuse. De même, lorsque, à Sparte, Ménélas offre trois chevaux à Télémaque, celui-ci ne peut accepter : « Je ne puis emmener des chevaux en Ithaque; c'est un luxe qu'ici j'aime mieux te laisser, car ton royaume, à toi, est une vaste plaine, qui porte en abondance le trèfle, le souchet, l'épeautre, le froment et la grande orge blanche. Ithaque est sans prairies, sans places où courir : ce n'est qu'île à chèvres !» ( $\delta 601-606$ trad. V. BÉRARD).
} 
d'autres ont des domaines beaucoup plus riches que lui. On comprendrait dès lors que, dans la crise économique que traverse la Grèce de la fin du IXe s. et du début du VIIle s., parce que le sol n'arrive plus à produire assez pour nourrir une population plus nombreuse, l'intelligence active et pratique d'Athéna ait paru indispensable. À l'origine déesse protectrice de la maison, mais qui, à l'inverse d'Héra, en est sortie, Athéna allait pouvoir aider les Grecs dans toute entreprise où l'habileté manœuvrière allait être indispensable. Son importance dans le panthéon grec ne pouvait s'en trouver qu'augmentée.

On en arrive ainsi à la conclusion suivante, hypothétique certes, mais qui semble en gros pouvoir être considérée comme probable. Le culte d'Athéna existait certainement en Grèce dès l'époque mycénienne, mais l'unique mention qui est faite de la déesse sur les tablettes ${ }^{75}$ pourrait indiquer que son culte était limité. Peut-être, éventuellement, faut-il la trouver sous l'appellation

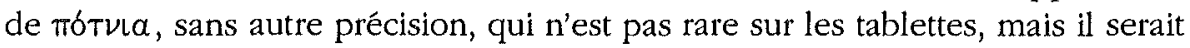
dangereux de rien en conclure. Quoi qu'il en soit, il s'agissait très probablement d'une déesse gardienne de la maison, ce n'est que plus tard, à l'époque de la composition de l'Iliade et surtout de l'odyssée, qu'Athéna prendra le rôle essentiel qui sera désormais le sien. Son importance croissante, comme d'ailleurs celle d'Apollon, est liée à des circonstances socio-économiques.

Universitê de Liège

Paul WATHELET

Philologie Classique

Place du 20-Août, 32

B - 4000 LIËGE

75 À Cnossos KN V $52+52$ bis +8285 , 1. La ligne 2 porte la mention d'e-nu-wa-ri-jo (Enyalios), pa-ja-wo-ne (Péon) et po-se-da-o-ne (Poseidon). On ne peut tirer de la mention d'Enyalios avec Athéna un éventuel caractère guerrier de la déesse, puisqu'elle est aussi en compagnie de Péon et de Poseidon. 